\title{
Korea's National HRD Reform Strategy for SDG
}

\author{
In-Pyo Hwang* \\ Professor at the National Human Resources Development Institute (NHI) under the Ministry of Personnel \\ Management (MPM)
}

*Corresponding Author: In-Pyo Hwang, Professor at the National Human Resources Development Institute (NHI) under the Ministry of Personnel Management (MPM)

\begin{abstract}
How should we respond to future changes in the public HRD area? The key role of a government will be developing and managing human resources that are prepared for the future.

The focus of the Korean government's HR policy is shifting from seniority, homogeneous civil service culture, rotation, simple wage system, and performance evaluation towards balance, openness, competition, expertise strengthening, goal-oriented system, and comprehensive evaluation.

As such, this paper addresses; Why Korea needs Sustainable Development Goal (SDG) for the future?; Strategy for future-oriented capability development system; Strategy for strengthening future-oriented policy and communication capabilities; Strategy for advancing knowledge ecosystem based on social learning;
\end{abstract}

Lastly, some implication as being summarized those issues are shown.

Keywords: HR policy, HRD strategy, SDG (sustainable development goal), public civil service

\section{INTRODUCTION}

A new paradigm is emerging in administration. Traditional administrative theories pursue a big government focusing on bureaucracy, principle, management mechanism, and government-led public projects, while new public management theories pursue a small government focusing on new liberalism-based administration, market mechanism, and outsourcing to the private sector. However, today's new public service theories pursue a government with a proper size focusing on community based administration, trust, process, participation mechanism, and cooperation with citizens and enterprises. The role of a government was rowing and steering in the past, while the role of a government is co-creating nowadays.

Then how should we respond to future changes in the public HRD area? The key role of a government will be developing and managing human resources that are prepared for the future. In other words, nurturing future-oriented public leaders will become very important. For a nation to join the ranks of advanced countries, the key decision makers in the government should be equipped with advanced leadership that eagerly seeks advancement of the system and maximization of individual and organizational capabilities.

\section{IMPORTANCE OF PUBLIC HRD}

Now is the time for a paradigm shift in public HRD. We should strengthen capabilities through recruitment and performance management of outstanding human resources. Capability strengthening in the public sector is not just a waste of money but a valuable asset where investment should be made. To ensure effective implementation of administrative reform, a consensus on national development should be made based on self-reflection and internalization of new values and ideologies. Improvement of capabilities and learning abilities of new talents will lay the foundation for continuous implementation of reform. An individual should make a change, participate in reform, enhance awareness, rebuild culture, and improve core capability and expertise in order to strengthen competitiveness in the public sector. For this, a massive shift in HRD strategy should take place.

However, there is still a room for improvement: a manager who can't answer a question about policy direction, saying that he didn't receive a consulting report yet; a manager who failed to identify the 
characteristics of his close colleagues after spending a year in the department; everyone is silent in a meeting except for a newly recruited employee; a leader who spends a whole hour chitchatting with an employee during an official meeting; and a manager who likes to give a speech or lecture that is irrelevant to the agenda of the meeting.

\subsection{HR Policy and HRD Strategy}

The focus of the Korean government's HR policy is shifting from seniority, homogeneous civil service culture, rotation, simple wage system, and performance evaluation towards balance, openness, competition, expertise strengthening, goal-oriented system, and comprehensive evaluation. This is because there have been concerns: whether simple seniority and performance evaluation can motivate core talents; whether seniority and ranking system can propel creativity in young people; and whether an underperformer can guarantee a rosy future of an organization.

Michael Kocken(2014) argued in his paper on the productivity of HRD that there are eight reasons why Korea has relatively low organizational productivity among OECD countries: rigid organizational structure and hierarchy; lack of communication; too much use of cellphone and SNS during work; stress and hangover from heavy drinking; more emphasis on format not on contents; low capabilities of the newly recruited talents; pretending to be busy at all times; and lack of efficiency in completing a task.

We are living in the era of the Fourth Industrial Revolution, and there is so much uncertainty due to complicated and rapid changes that it is difficult to even predict a year later.Gary Hamel argued in his book The Future of Management, "Trouble is, obedience, diligence, and competence are becoming global commodities. You can buy these human capabilities just about anywhere in the world, and in places like India and China, they can be bought for next to nothing." He stressed that passion and creativity is the key for creating high value-added products.

Infinite competition derived from exponential changes in politics, economy, and globalization is something that we were not able to imagine in the past. To survive this competition, we need to produce better products and differentiate services from our competitors. What is most important for achieving this goal is efficient HRD and management that maximizes an individual's potential in the organization.

How can we respond to various changes and demands in this era? The six future HR strategies include differentiation, role \&responsibility, talent management, attrition management, diversity, and work \& life balance.

For differentiation, we need to pursue balance between differentiation and seniority, financial compensation, and preferential treatment for the experienced. For role \&responsibility, we need to think about strict performance management, strengthened role of manager, manager's intuition, experience, effort, and compensation for manager. For talent management, we need to focus on core talent management, attention to young, female, and minority talents, and succession plan. For attrition management, we should consider distinguishing good attrition from bad attrition, opportunity and consideration, and balance between individual and society. For work \& life balance, which is emerging as an important issue nowadays, we need to think about how to find balance between an individual's work and life.

However, it is not easy for a public organization to take flawless responsibility for HRD. First of all, it is difficult to satisfy all stakeholders who have different interests in performance evaluation. It is also not easy to quantitatively and visibly measure an organization's performance related to HRD. Sometimes it is impossible to measure economic feasibility, efficiency, and effectiveness of HRD even when an organization achieved them all. Nevertheless, public organizations continue to emphasize performance evaluation as a way of defining an organization's sense of responsibility in HRD.

Whether it is possible to quantitatively measure HRD in an organization is always an issue, as no evaluation can measure all aspects of human relations. However, there are now more tools for quantitative measurement. Therefore, it is true that adequate design and execution of an evaluation can enhance the quality and efficiency of public service.

\section{NEW NATIONAL HRD REFORM STRATEGY: 'NATIONAL HRD GRAND DESIGN 100' PROJECT}

\subsection{Why Korea needs Sustainable Development Goal (SDG) for the Future?}

As mentioned above, creative, open, and convergent talents who can provide clear vision and lead 
changes became important amid rapid changes in the environment. As we are living in the era of the Fourth Industrial Revolution, which is characterized by VUCA (Volatility, Uncertainty, Complexity, Ambiguity), nurturing human resources equipped with expertise has become a priority.

In addition, Capitalism 4.0. stresses human-oriented economic and social system where society and nation take responsibility of helping people who are left behind. Contribution to public interest and community development through pursuing social value, responsibility, human rights, safety, and consideration of the underprivileged has become the basic principle of state affairs. As a result, a new mission of realizing social value and contributing to the community has emerged.

HRD strategy is the core element that decides the fate of a nation or an organization. The world is now in the war for talent. Public HRD strategy should be established with fundamental principle and direction in order to respond to the demand from the society.

Against this backdrop, National Human Resources Development Institute (NHI) came up with "National HRD Grand Design 100" as part of Sustainable Development Goal (SDG). NHI has led national development for the past 70 years as a cradle for national HRD since its inception in 1949. Now there is a need to newly establish a milestone of national HRD for the next 30 years. In preparation for its 100th anniversary, NHI developed a new vision of "good HRD and warm NHI" and a new mission of "cradle for government officials who can lead the way into a new era for the Republic of Korea". Its five action plans include "base camp for government official training", "academy for administrative leaders", "center of R \& D", "hub for global leadership", and "community based platform". Core values that support the vision include excellence, creativity, collaboration, and commitment.

\subsection{Strategy for Future-Oriented Capability Development System}

\subsubsection{Establishment of "Capability Development Center" for Future Core Leaders}

Amid future changes, NHI planned to continuously develop and systemize core capabilities, build customized capability development model, and share and spread the model globally. The center is expected to elevate the status of Korea through improving responsiveness to the changes, strengthening HRD capabilities, fostering outstanding talents, enhancing national competitiveness, and sharing HRD knowhow with the international society.

<Figure 1> shows core capabilities and education model reform in response to the future changes. Through analyzing roles and responsibilities of public officials in the future, NHI is going to identify core capabilities for each grade and redesign education programs based on newly defined priorities.

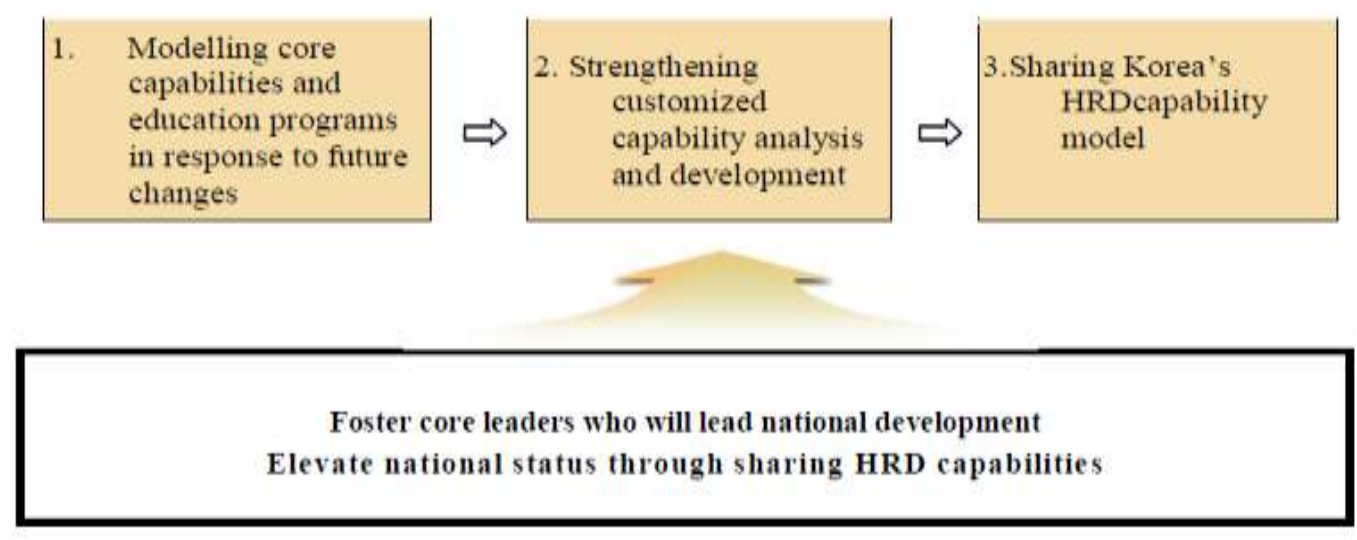

Figure1: Core capabilities and education model reform

NHI is also planning to provide systematic consulting service based on capability ecosystem through standardizing capability education operation system. The service will form a cycle of capability diagnosis, analysis, modelling, consulting, accumulation, and feedback for educational organizations. $\mathrm{NHI}$ is going to play a key role as a capability education academy for public HRD organizations under the government, and improve the design and operation of capability based education systems. NHI will set up mid to long term capability education strategies that are customized to each department and ministry in the government, leading the implementation of national leadership and capability education strategies. 


\subsubsection{Construction of Public HRD Knowledge Archive}

$\mathrm{NHI}$ is planning to construct public HRD knowledge archive for integrated management and sharing of national resources on public HRD. First, it will set up a system that can systematically integrate and manage HRD knowledge generated inside and outside of NHI. Second, it will hold HRD contest and competition in order to develop and accumulate quality education contents. Third, it will construct an archive with easy access, allowing anyone to utilize and share HRD related knowledge and information through the system.

The system will allow integrated utilization of HRD knowledge and resources, maximizing synergy effect in the public HRD domain. Free distribution, utilization, and processing of public HRD information will create new knowledge by escalating the quantity and quality of HRD resources. The archive will also work as national knowledge bank, information hub, and think tank for public HRD, contributing to fostering excellent public human resources in the future.

\subsubsection{Foundation of Policy Case Study Hub}

NHI is planning to become the best policy case study hub in Asia through shifting its education paradigm from lecturing to participating and problem-solving. It will foster utilizing case studies in the education program to enhance policy analysis and problem-solving capabilities and creativity of public officials. This paradigm shift is expected to increase effectiveness of education immensely, as it provides practical examples that can be applied immediately. By sharing case study education programs with educational organizations, universities, and overseas HRD organizations, NHI is expected to become a case study hub in public HRD.

To this end, NHI is going to continuously develop policy cass. We will collect preliminary materials on pre-policy stage following policy quality management manual. In the process, we will pursue "one-source multi-use" principle, and particularly focus on the latest policy cases. We will track down and analyze policy cases for a long time. This is because we should consider social and environmental aspects surrounding a policy. At times, a single policy requires long term trace and analysis. We will also rewrite existing and new cases according to various goals and methods of education.

NHI is going to establish comprehensive database on domestic and international policy cases. For this, we will categorize existing cases according to areas and types of policy. We will also collect and manage policy cases, relevant materials, and multimedia resources that can be used in education programs. We will also provide feedback on policy changes and case studies from public servants for continuous management of policy cases that can be used as education materials.

NHI is going to spread policy case study education. First, we will translate the best policy cases in Korea so that it can be used for overseas public official's training programs. The cases will be shared with overseas public HRD organization. Second, we will introduce new education methodology which facilitate problem-solving through field trips. Third, we will also consider providing various education programs through outsourcing and connecting the programs to a degree course. Fourth, we will secure quality professors for policy case study education. We will try to hire retired public servants, share professors with other educational organizations, and utilize university professors in their sabbatical years.

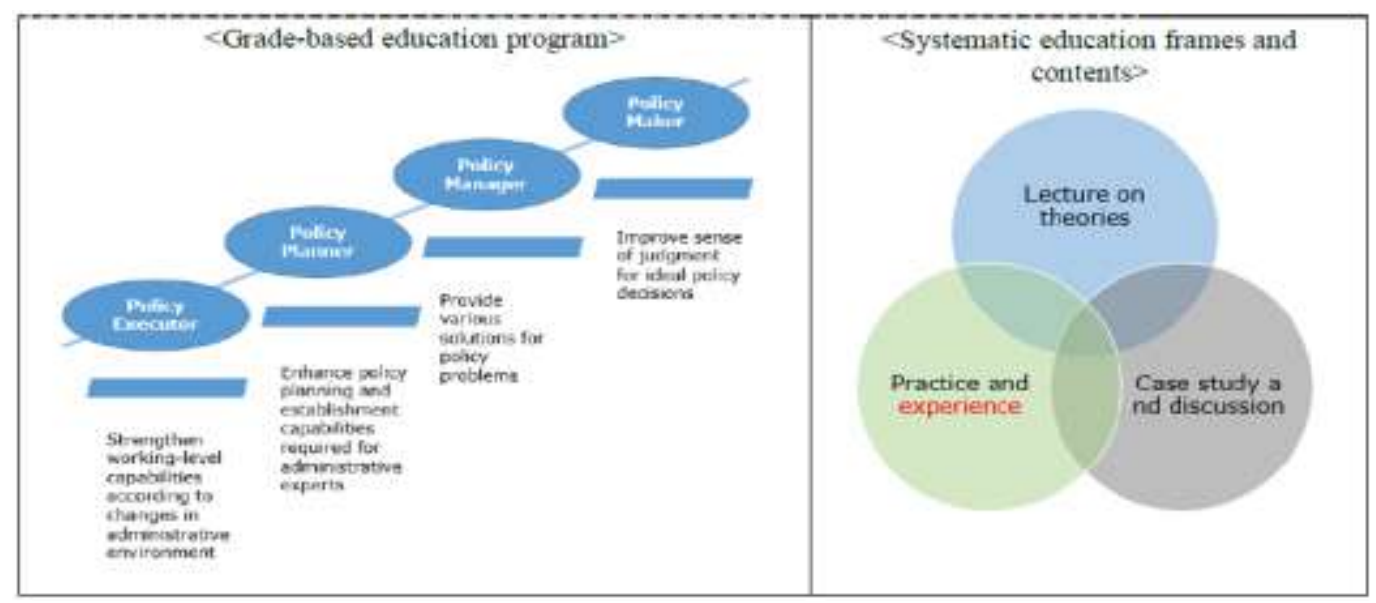

Figure2: Policy capability improvement program 
Fifth, we will establish professional education course for strengthening policy capabilities. As shown in <Figure 2>, new methodologies such as grade-based program, on the job training, case study, and discussion will be revitalized. Sixth, we will introduce policy education certificate system for evaluating the quality of policy education. The system will set up educational goal and standard for policy education, and evaluate the level and contents of policy education provided by educational organizations. Seventh, we will construct a cooperation system with public HRD educational organizations in Asia and advanced nations in the world. We will share the best policy cases to overseas organizations and introduce overseas policy cases to Korean public officials. Domestic policy cases will also be utilized in overseas public servants' programs in order to provide customized policy cases and educational methodologies for each nation.

\subsection{Strategy for Strengthening Future-Oriented Policy and Communication Capabilities}

\subsubsection{Implementation of "National Affairs CEO Forum with Global Scholars"}

NHI is planning to hold a forum with global scholars and leaders. Regular implementation of "National Affairs CEO Forum" will gather experts in various domain together to discuss global issues and solutions. Global scholars will be invited to attract government officials and CEOs of public organizations. The result of the forum will be announced as "National Affairs CEO Forum Declaration", and the members of the forum will be continuously managed as think tank.

The members of the forum will include decision makers of national policy (National Affairs CEOs) such as law makers, ministers, vice ministers, CEOs of public organizations, global scholars who can lead global trends, the Nobel Prize laureates and nominees, and CEOs of international organizations and NGOs in various domains.

\subsubsection{Opening of "Policy Explanation and Communication" Program for Better Communication with the Public}

NHI opened "Policy explanation and communication" program to better communicate with the public and to enhance policy explanation capability. Public servants have an obligation of policy explanation in addition to national defense, labor, education, and tax payment. To fulfill the obligation of policy explanation, social sensibilities should be enhanced through a new education program.

The program is designed to strengthen public official's policy announcement and explanation capabilities and to better communicate with the public. We are planning to expand the program by newly opening policy explanation and communication program that is differentiated for each grade.

\subsubsection{Establishment of "Master of policy" Program for Theory and Practice}

It is urgent for Korean public officials to strengthen policy establishment and management capabilities in response to changes in administrative environment such as the public's participation in policy making and complicated conflicts related to policies. In particular, conflict management education should be prioritized in order to prevent conflicts and disputes that inevitably take place in the process of policy implementation.

To this end, NHI is planning to operate Advanced Management Program. We are going to emphasize how to analyze conflicts and their impacts on policy establishment process, how to communicate, negotiate, mediate in the policy implementation process, and how to apply the relevant laws and regulations in the process. In addition, theories and case studies of conflict management, field trips, study groups for advanced research, and presentation of the final result will be included in the program.

The program will be expanded to become a certificate program for experts through collaboration with prestigious universities and the private sector in the future. The program will help public officials maintain their professional techniques in managing conflicts through providing up to date information in the domain.

\subsubsection{Operation of "Creative Policy Improvement" Program in Preparation for Changes in Future Industry}

The disruptive technologies of the Fourth Industrial Revolution are estimated to shift overall social paradigms and bring about changes in social structure. However, Korea is lagging behind the competitors in terms of education, technology, law and order, infrastructure, and labor. As nations and companies that lead the Fourth Industrial Revolution are expected to take a lead in the global 
economy, there is an urgent need for developing the relevant capabilities and for coming up with preemptive action plans through cooperation between the public and the private sector.

Therefore, a full package program should be developed and operated in order for public officials to understand rapidly changing environment, to predict possible problems, and to prevent problems in advance. We will provide a tool kit such as systems thinking for future estimation, analysis, understanding, and policy development to ensure integrated problem solving. Customized education for reasonable analysis, estimation, and practical problem solving programs will enhance effectiveness and utilization. The program will focus on practice and experience in order to strengthen understanding and utilization of the latest trends and core technologies.

The program is designed to improve responsiveness and competitiveness of the government through improving creative problem solving capabilities of public servants in various domains and leading future-oriented public HRD education.

\subsection{Strategy for Advancing Knowledge Ecosystem Based on Social Learning}

\subsection{1. "The Government E-Learning Platform" to Realize "Open Campus in My Hands"}

NHI advanced smart learning platform to provide customized education service for individuals at anytime and anywhere by utilizing various materials inside and outside of the government. The previous platform focused on PC and Mobile, and provided lecturer-oriented contents; while the new platform which was opened in January 2018 focus on advancement of mobile service that provide customized contents allowing communication and collaboration between a lecturer and a learner.

Through such advancement, the Government E-learning Platform came to be equipped with functions of knowledge production and storage based on active participation and collaboration of the learners instead of simply providing one time learning experience. NHI is planning to continuously revitalize the Government E-learning Platform by providing quality learning that meets the needs of learners and by actively promoting the function of the platform in the future.

This learner-oriented e-learning platform enables communication, collaboration, informal learning, and customization of the latest HRD contents. We are aiming at stabilizing main functions through recommendation service, social learning service, utilization of public education materials, and advancement of mobile service. E-HRD conference will also be held to share the case with domestic and overseas public officials.

\subsubsection{HRD 4.0: Sophistication of Knowledge Ecosystem based on Social Learning}

NHI will make an utmost effort to create knowledge ecosystem that allows knowledge production, sharing, utilization, and reproduction through social based collaborative learning environment where public officials' experiences and knowhow can be accumulated, shared, and utilized effectively. The platform will accumulate large size of information in various domains that will be shared among public officials to improve work efficiency and collaboration. Such platform will also provide customized learning service that meets the needs of an individual.

This mobile-oriented platform will provide social learning tools for collaborative education and create knowledge sharing environment so that learners can produce and utilize knowledge at anytime and anywhere utilizing mobile devices such as smart phone. Mileage compensation system for knowledge production, sharing, utilization, and reproduction will be introduced to revitalize social learning and to encourage evolvement of knowledge accumulated in e-learning platform and community.

\section{CONCLuSion}

The reasons why public HRD failed to lead a new trend or adapt to one in the face of changes in the new era are relying too much on the experience, trusting the existing strength too much, and deluding that it is already familiar with a new trend. Despite the emergence of new knowledge ecosystem and environmental changes such as the Fourth Industrial Revolution, public HRD failed to implement innovative strategies and build up preemptive minds.

The reasons why public HRD failed to manage policy stake holders properly are work-oriented approach or behavior, lack of recognition of interrelations with people outside the organization, obsession of administrative process that is irrelevant to policy, and lack of understanding or ignorance of policy.

Governments of today are trying to conduct reform and establish a system for fostering public human resources in respond to the coming future. It is critical for the Korean government to construct sustainable and innovative HRD system that can overcome the limitations of existing HRD strategies and policies. 
In this regard, implementation of "National HRD Grand Design 100" as a part of new national HRD reform strategy is very meaningful. It would be very timely and proper to establish a vision for SDG, create future-oriented capability development system, strengthen future-oriented policy and communication capabilities, and advance knowledge ecosystem through social learning.

People who successfully lead future changes are the ones who courageously stand up as an agent of change, trust various stake holders, and are eager to share and spread changes. They are the ones who learn from their mistakes, reduce uncertainty in the environment, and provide clear vision. When these people who are equipped with innovative minds step up to emphasize the importance of public HRD strategy for future development, public officials will be able to adapt well to the new administrative environment and play a valuable role in the era where citizens represent a nation.

\section{REFERENCES}

[1] Back, G.B. (1994), Organizational Behavior, Bobmun Publishing, Seoul.

[2] Hamel, Gary (2007), The Future of Management, Harvard Business School Press.

[3] Hwang, I.P. (2018), The Future Strategy for Human Resource in Public Sector, Public HRD, NHI, 81(Spring): 8-12.

[4] Hwang, I.P. (2016), strengthening for HRD Potential through Training and Education, Public HRD, NHI, 74(spring): $18-23$.

[5] Hwang, I.P. (2015), The Role of Public HRD on Social Change, Public HRD, NHI, 72(Autumn): 15-18.

[6] Hwang, I.P. (2013), Activating of Evidence-based Education Using Action Plan), Public HRD, NHI, 65(Winter): 57-61.

[7] Hwang, I.P. (2006), Change of Leadership Paradigm with Mid-managers, Public HRD, NHI, 47(Spring): 62-75.

[8] Kanter, R.M. (1996), World Class Leaders: The Power of Partnering, In Hesselbein, F., Goldsmith, M., \&Beckhard, R. (ed.), The Leader of the Future, San Francisco, CA: Jossey-Bass, 89-98.

[9] Kettner, P.E. (2002), Achieving Excellence in the Management of Human Service Organization, MA: Allyn \& Bacon

[10] Kocken, Michael (2014), Why Korea Shows Low Labor Productivity in Organization, The Sawon blog.

[11] Kotler, P. (2005), Marketing Management, Englewood Cliffs, NJ: Prentice Hall.

[12] Lee, S.H. (2001), Leadership Theories and Research Activities in Korea, Korean Academy of Organization and Management, 24(2): $1 \sim 40$.

[13] NHI (2017), 'National HRD Grand Design 100’ Project, NHI, Seoul.

[14] NHI (2017), 'National HRD Grand Design 100' Project- Action Plan I V, NHI, Seoul.

\section{AUTHOR BIOGRAPHY}

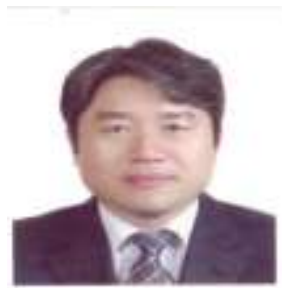

National Human Resources Development Institute (NHI), Ministry of Personnel Management (MPM), Korea.

Ph.D. University of York, UK, MA ChungAng University, Korea, Post-doctorate University of Birmingham, UK.

The work currently is focused on leadership, managerial strategy and HRD/HRM after joined in the NHI on 2005. Senior specialist, Korea Institute of Science and Technology(KIST); Professor, Kwangju Women's University, Korea; Visiting professor, Wharton SchoolUPenn and California State University, Fresno, USA.

Books- 'Leadership in Public Sector' 'Organizational Change and Value' 'Managing for Job Performance'.

Citation: In-Pyo Hwang. "Korea's National HRD Reform Strategy for SDG " International Journal of Managerial Studies and Research (IJMSR), vol 7, no. 10, 2019, pp. 26-32. doi: http://dx.doi.org/10.20431/ 2349-0349.0710004.

Copyright: () 2019 Authors. This is an open-access article distributed under the terms of the Creative Commons Attribution License, which permits unrestricted use, distribution, and reproduction in any medium, provided the original author and source are credited. 